\title{
Effects of neonate size and food concentration on the life history responses of a clone of the hybrid Daphnia hyalina $\times$ galeata to fish kairomones
}

\author{
TINEKE REEDE \\ Department of Aquatic Ecology, University of Amsterdam, Kruislaan 320, 1098 SM Amsterdam, The Netherlands
}

\section{S U M M A R Y}

1. The influence of fish kairomones on the life history parameters of a clone of the hybrid Daphnia galeata $\times$ hyalina was measured in laboratory experiments with small and large neonates at one food concentration. The influence of fish kairomones on neonates of the same size at two food concentrations was also investigated.

2. Neonate size (maternal investment per egg) changed the influence of fish kairomones on life history parameters. Daphnids that were small at birth showed greater differences between fish (kairomones present) and no-fish (kairomones absent) treatments for age at maturity and number of eggs in the first clutch than animals that were large at birth. On the other hand, animals that were large at birth differed more in size at maturity between fish and no-fish treatments than small neonates.

3. The food concentrations used in the experiments $\left(0.25-0.75 \mathrm{mg} \mathrm{Cl}^{-1}\right)$ hardly affected the influence of fish kairomones on the life history characteristics studied.

4. The effect of fish kairomones on the size of neonates from the first clutch was similar for large and small daphnids. In the no-fish treatment significant differences were found between small and large neonates for age at maturity and number of eggs in the first clutch. In the fish treatment size at maturity differed significantly between small and large neonates.

5. In life history experiments, neonate size has to be taken into account when measuring the effects of fish kairomones because size at birth influences the results.

\section{Introduction}

Food concentration is one of the most important factors influencing life history parameters in animals. In Daphnia, for example, food concentration influences maternal investment which is reflected in the size and number of offspring. Well-fed Daphnia produce large clutches of small neonates, while with less food they produce small clutches of large neonates (Glazier, 1992; Guisande \& Gliwicz, 1992), although some studies have reported the opposite relationship (Urabe, 1988; Tessier \& Consolatti, 1991). Glazier (1992) found that at very low food concentrations, size at birth became smaller again. Thus, the disparity between these studies might be due to a hump-shaped reaction norm for egg size in Daphnia with a decrease in neonate size at very low food concentrations (Glazier, 1992; Ebert, 1994). Neonate size is an important life history trait because it is of influence in later life. For instance, a positive relationship between egg size and size at first reproduction was shown by Lampert (1993). Furthermore, it has been shown that large juveniles survive longer under poor food conditions than small ones (Cowgill, Williams \& Esquivel, 1984; Tessier \& Consolatti, 1989). Large young are also better able to withstand attacks by invertebrate predators, that are more abundant in the absence of vertebrate predators. On the other hand, smaller sized daphnids are less visible and thus will have higher chances 
of survival and reproduction in times of heavy predation by fish.

Predatory fish influence Daphnia populations not only by selective feeding, but also because they produce kairomones which cause Daphnia to mature at smaller sizes and younger ages and to produce larger first clutches (Macháček, 1991; Stibor, 1992). Juveniles born in the presence of fish are smaller (Reede, 1995), thus making them less vulnerable to predation but more vulnerable to starvation (Cowgill et al., 1984). The extent of the life history changes due to fish kairomones depends on the kairomone concentration (Reede, 1995) and on genetic differences between clones (Reede \& Ringelberg, 1995). The reaction to fish kairomones also depends on the juvenile stage at which an individual is exposed (Macháček, 1995). Neonate size may also play a role in kairomone sensitivity.

Although food concentration and fish kairomones can both influence the life history of Daphnia, the changes induced by kairomones might not be the same at all food concentrations. A study by Macháček (1991) revealed no effect of food concentration, but Reede \& Ringelberg (1995) observed that fish kairomones increased the intrinsic rate of increase less at a high than at a low food concentration. The effect of kairomones from an invertebrate predator was influenced by food concentration in a study by Barry (1995). He found that in Daphnia carinata King, food supply had a small but significant effect on relative crest height in the presence of kairomones from its predator, Anisops gratus Hale. On the other hand, Tollrian (1995) found that the formation of neckteeth in Daphnia, in response to a chemical released by Chaoborus, was not affected by food concentration. However, Parejko \& Dodson (1991) reported that responses to kairomones from Chaoborus were influenced by food concentration.

The role of neonate size and food concentration in the reaction to fish kairomones has not been investigated hitherto. Size at birth affects sensitivity to several chemicals (Enserink, Luttmer \& Maasdiepeveen, 1990; Naylor, Bradley \& Calow, 1992; Hanazato \& Dodson, 1995) and it might also affect sensitivity to chemicals produced by fish. The purpose of this study was to examine how neonate size and food concentration influence life history parameters in the presence of predatory fish. Life table experiments were performed in the laboratory with a clone of the hybrid Daphnia hyalina (Sars) $\times$ galeata (Leydig).

\section{Materials and methods}

\section{Experimental animals}

Life history experiments were performed with a clone (clone O2, collection of the Department of Aquatic Ecology, University of Amsterdam) of the hybrid Daphnia galeata $\times$ hyalina originating from Lake Maarsseveen. This clone had been cultivated in the laboratory for many generations prior to the experiments.

\section{Experimental design}

A flow-through system, as described by Reede \& Ringelberg (1995), was used for life history experiments. Individual animals were kept in small tubes (15 ml) with gauze bottoms $(150 \mu \mathrm{m}$ mesh size) through which algae could pass freely but daphnids could not. Seven tubes in a cuvette represented one experimental unit. The culture medium in the cuvettes was refreshed with media from reservoir bottles every $4 \mathrm{~h}$, while stocks in the reservoir bottles were renewed daily. In this way, food concentration in the tubes was kept relatively constant throughout the day (variation was less than $25 \%$ ). Cuvettes were placed in a water bath to keep temperatures constant at $15 \pm 1.0{ }^{\circ} \mathrm{C}$. The positions of tubes within the same treatment were randomly changed every other day. Redistribution took place within as well as between cuvettes in the same treatment.

Two experiments were carried out. In experiment 1 , the influence of fish kairomones on large and small neonates was investigated. Large neonates were produced by mothers reared at low food concentration, whereas small neonates were from mothers reared at high food concentration. Experiment 2 was designed to determine the effect of fish kairomones on life history characteristics at different food concentrations. In this experiment, neonate size and maternal investment was the same for the experimental animals.

Each experiment was started with female neonates less than $18 \mathrm{~h}$ old. Water used in the experiments originated from the hypolimnion of Lake Maarsseveen. Hypolimnetic water does not contain fish kairomones, because fish usually do not go below the thermocline. 
Nevertheless, the water was filtered continuously through a sand filter to facilitate bacterial breakdown of any traces of kairomones present. Before use, the water was filtered through a $0.2 \mu \mathrm{m}$ membrane filter. Algae (Scenedesmus acuminatus (Lagerheim) Chodat) were grown in Woods Hole MBL (Guillard, 1975) in continuous culture. Only log-phase cells were used as food in the experiments. Food concentration was measured with a Coulter counter and expressed as $\mu \mathrm{m}^{3} \mathrm{ml}^{-1}$. A calibration curve, $\mu \mathrm{m}^{3} \mathrm{ml}^{-1} v \mathrm{mg} \mathrm{Cl}^{-1}$, was obtained using a carbon analyser (model 700 TOC analyser, O.I. Analytical).

Water containing fish kairomones was taken from an aquarium, which housed ten $0+$ perch (Perca fluviatilis (Linnaeus)) of about $4 \mathrm{~cm}$ length and was filled with 201 of hypolimnetic water from lake Maarsseveen. This water was also filtered through a $0.2 \mu \mathrm{m}$ membrane filter before use in the experiments. One-third of the water in the aquarium was refreshed every day and faeces were removed simultaneously. The perch were caught in Lake Maarsseveen and were fed Chironomus larvae. To expose Daphnia to fish kairomones, experimental animals were grown in water containing two-thirds hypolimnion water and one-third water from the aquarium. This concentration of fish kairomones gave an intermediary to high response in Reede (1995). Henceforth, experimental treatments in which kairomones were used will be called fish treatments, and those without kairomones will be called no-fish treatments. The experiments were continued until neonates from the first clutch were released. In each treatment twenty-eight animals were used initially, but in some treatments between one and four animals accidentally died.

In experiment 1 , grandmothers and mothers (generation 1) of experimental animals (all third clutch daughters) were grown at either a low food concentration $\left(0.1 \mathrm{mg} \mathrm{C}^{-1}\right)$ or a high food concentration (1.0 $\left.\mathrm{mg} \mathrm{Cl}^{-1}\right)$, yielding large and small neonates, respectively. The experimental animals (generation 2) were all provided with $0.25 \mathrm{mg} \mathrm{Cl}^{-1}$ of $S$. acuminatus in the presence and absence of kairomones, resulting in four experimental situations. In experiment 2 , neonates from one cohort (the third clutches of ten mothers) of which the mothers had all been given the same food concentration $\left(0.25 \mathrm{mg} \mathrm{Cl}^{-1}\right)$ were divided over four treatments. Experimental animals were grown at two food concentrations, 0.25 and $0.75 \mathrm{mg} \mathrm{Cl}^{-1}$, and at each food concentration there was a fish and a no-fish

(C) 1997 Blackwell Science Ltd, Freshwater Biology, 37, 389-396 treatment. Length at birth, age and length at maturity, number of eggs and the length of the neonates from the first clutch were determined for each individual. The first clutch is most important in determining $r$, especially when fish predation reduces the life expectancy of adults. Time and size of the first clutch are very important in determining the intrinsic rate of increase. Therefore, animals were allowed to produce one clutch per animal, after which population growth rates were calculated according to the Euler equation:

$$
1=\Sigma \mathrm{e}^{-r x} l_{x} m_{x}
$$

where $r=$ intrinsic rate of increase for the population, $x=$ age class in days, $l_{x}=$ probability of surviving to age $x$, and $m_{x}=$ age-specific fecundity. Since $r$ cannot be isolated on one side of the equation, iterative calculations had to be performed. However, this left us with only one value for $r$ per data set whose variance could not be calculated. A variance was generated therefore by applying the jack-knife method described by Meyer et al. (1986). The values for $r$ after one clutch underestimate the results of $r$ calculated after several clutches.

Age at maturity was defined as the day on which the first eggs appeared in the brood pouch. Lengths were measured from the edge of the head, through the eye, to the base of the tail. Two-way ANOVAs were conducted to test for significant effects of food concentration, kairomone presence, and their interaction. Pairwise comparisons were carried out with the Student-Newman-Keuls test (Sokal \& Rohlf, 1981).

\section{Results}

Experiment 1: effect of neonate size and fish kairomones

Animals whose mothers and grandmothers were grown at a low food concentration were significantly larger at birth $(\mathrm{df}=58, P<0.001)$ than those whose parents were grown at a high food concentration $(0.71 \pm 0.005$ and $0.68 \pm 0.006 \mathrm{~mm}$, respectively). Thus, under low food conditions mothers invest more energy into individual eggs than at high food concentrations.

Two-way ANOVAs showed a significant effect of kairomone presence on the life history traits studied. Maternal investment (i.e. neonate size) had a significant effect on the age at maturity, size at maturity and 

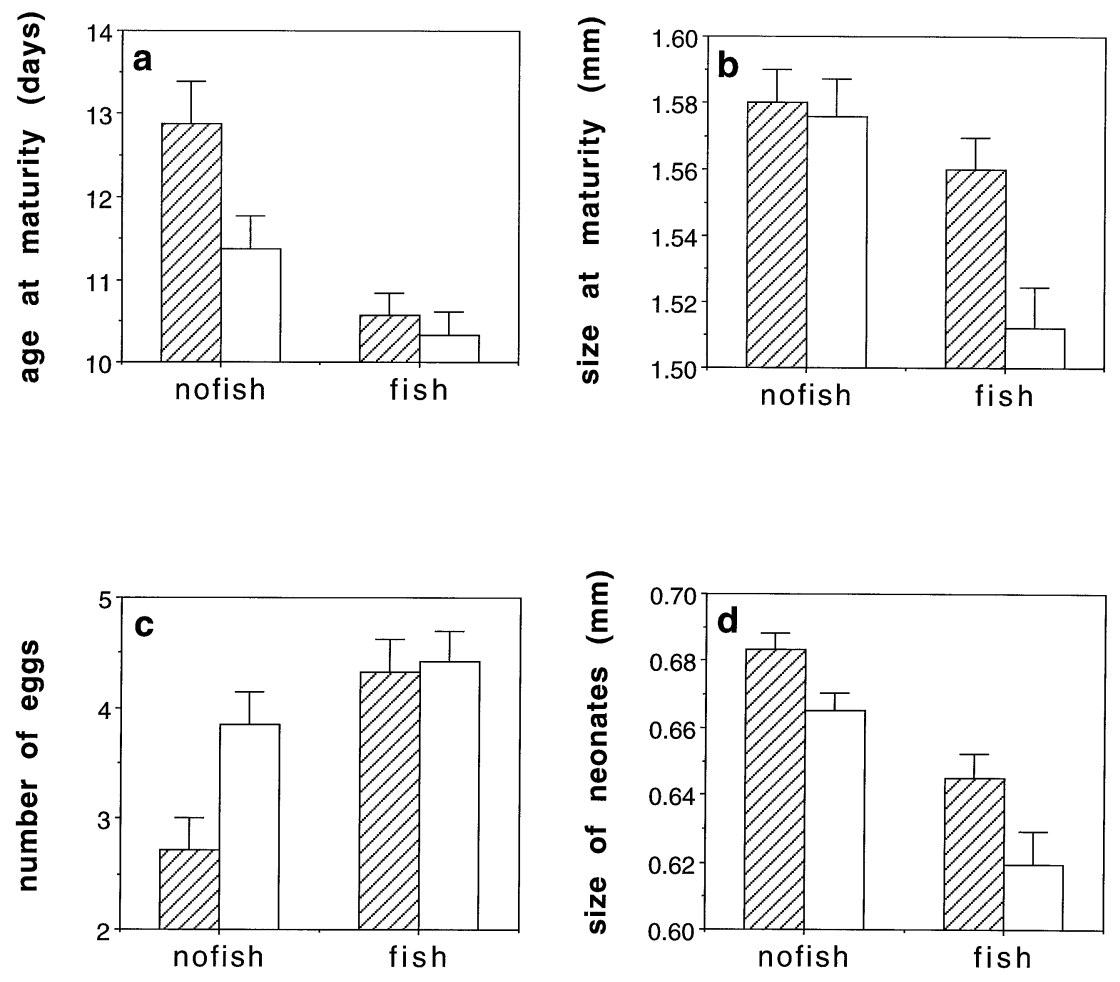

Fig. 1 (a) Age at maturity, (b) size at maturity, (c) number of eggs in the first clutch and (d) size of neonates from first clutch in experiment 1. Error bars represent SEs. Shaded bars: small neonates; open bars: large neonates.

Table 1 ANOVA table for life history traits in experiments 1 and 2. Neo, neonate size (experiment 1); food, food concentration (experiment 2)

\begin{tabular}{|c|c|c|c|c|c|c|c|}
\hline \multirow[b]{2}{*}{ Life history trait } & \multirow[b]{2}{*}{ Effect } & \multicolumn{3}{|c|}{ Experiment 1} & \multicolumn{3}{|c|}{ Experiment 2} \\
\hline & & $\mathrm{df}$ & $F$ & $P$ & $\mathrm{df}$ & $F$ & $P$ \\
\hline \multirow[t]{4}{*}{ Age at maturity } & Kairomone & 1 & 19.69 & $<0.001$ & 1 & 30.01 & $<0.001$ \\
\hline & $\mathrm{Neo} /$ food & 1 & 5.324 & 0.022 & 1 & 4.01 & NS \\
\hline & Interaction & 1 & 2.83 & NS & 1 & 0.881 & NS \\
\hline & Error & 99 & & & 105 & & \\
\hline \multirow[t]{4}{*}{ Size at maturity } & Kairomone & 1 & 10.83 & 0.001 & 1 & 21.79 & $<0.001$ \\
\hline & $\mathrm{Neo} /$ food & 1 & 5.87 & 0.017 & 1 & 4.34 & 0.043 \\
\hline & Interaction & 1 & 3.94 & NS & 1 & 0.333 & NS \\
\hline & Error & 99 & & & 105 & & \\
\hline \multirow[t]{4}{*}{ Number of eggs in first clutch } & Kairomone & 1 & 13.65 & $<0.001$ & 1 & 3.96 & NS \\
\hline & $\mathrm{Neo} /$ food & 1 & 4.37 & 0.042 & 1 & 26.35 & $<0.001$ \\
\hline & Interaction & 1 & 3.15 & NS & 1 & 0.014 & NS \\
\hline & Error & 99 & & & 105 & & \\
\hline \multirow[t]{4}{*}{ Size of neonates in first clutch } & Kairomone & 1 & 8.50 & 0.005 & & & \\
\hline & $\mathrm{Neo}$ & 1 & 0.569 & NS & & & \\
\hline & Interaction & 1 & 0.050 & NS & & & \\
\hline & Error & 69 & & & & & \\
\hline
\end{tabular}

the number of eggs in the first clutch. No interaction between food concentration and maternal investment was found, however (Table 1).

Pairwise comparisons showed several significant differences (Table 2, Fig. 1). Small neonates differed significantly between the fish and no-fish treatments for four out of five traits investigated here, while large neonates differed significantly for two out of five. Significant differences between small and large neonates in the fish treatment existed only for size at (C) 1997 Blackwell Science Ltd, Freshwater Biology, 37, 389-396 
Table 2 Matrices indicating significant differences at the 95\% level: *, significant; NS, not significant. AGEMAT, age at maturity; SIZEMAT, size at maturity; EGGSMAT, number of eggs in the first clutch; NEOSIZE, size of neonates in the first clutch; INTRINSIC, intrinsic rate of increase after one clutch. Experiment 1: $1=$ small neonates, no-fish; $2=$ small neonates, fish; $3=$ large neonates, no-fish; $4=$ large neonates, fish. Experiment 2: $1=$ high food, no-fish; $2=$ high food, fish; $3=$ low food, no-fish; $4=$ low food, fish

\begin{tabular}{|c|c|c|c|c|c|c|c|c|c|c|c|c|c|c|c|}
\hline & \multicolumn{3}{|c|}{ AGEMAT } & \multicolumn{3}{|c|}{ SIZEMAT } & \multicolumn{3}{|c|}{ EGGSMAT } & \multicolumn{3}{|c|}{ NEOSIZE } & \multicolumn{3}{|c|}{ INTRINSIC } \\
\hline & 1 & 2 & 3 & 1 & 2 & 3 & 1 & 2 & 3 & 1 & 2 & 3 & 1 & 2 & 3 \\
\hline \multicolumn{16}{|c|}{ Experiment 1} \\
\hline 2 & $*$ & & & NS & & & * & & & $*$ & & & $*$ & & \\
\hline 3 & NS & NS & & NS & NS & & * & NS & & NS & NS & & NS & NS & \\
\hline 4 & * & NS & NS & $*$ & $*$ & $*$ & * & NS & NS & $*$ & NS & * & * & NS & NS \\
\hline \multicolumn{16}{|c|}{ Experiment 2} \\
\hline 2 & * & & & * & & & NS & & & & & & NS & & \\
\hline 3 & * & * & & NS & NS & & * & * & & & & & $*$ & * & \\
\hline 4 & * & NS & * & $*$ & NS & * & * & * & NS & & & & NS & NS & NS \\
\hline
\end{tabular}

maturity. Surprisingly, the small neonates matured at a significantly larger size than the large ones (Fig. 1b). In the no-fish treatment significant differences between small and large neonates were present for age at maturity and number of eggs in the first clutch. The intrinsic rate of increase after one clutch (not shown in Fig. 1) was similarly affected by neonate size and fish kairomones, as was the number of eggs in the first clutch (Fig. 1c). In the no-fish treatment, $r$ for the small neonates was $0.066 \pm 0.0144$ (mean $\pm \mathrm{SE}$ ), and for the large neonates was $0.099 \pm 0.0096$. In the fish treatment, $r$ for small neonates was $0.114 \pm 0.0089$ and for large neonates was $0.113 \pm 0.0087$. Only the difference between the no-fish and fish treatments of the small neonates was significantly different at $P<0.05$.

\section{Experiment 2: effect of food concentration and fish kairomones}

Two-way ANOVAs showed a significant effect of kairomones on age at maturity and size at maturity, but not on clutch size. Both food concentration and the presence or absence of kairomones had significant effects on size at maturity, but no interaction effect was found. Only food level influenced the number of eggs (Table 1).

Significant differences between fish and no-fish treatments existed for the age at maturity and size at maturity at both food concentrations (Fig. 2a,b), but not for the number of eggs in the first clutch and $r$. Between the food concentrations, significant differences were found for the age at maturity, number of eggs in the first clutch and $r$ in the nofish treatment, but only for the number of eggs in the fish treatment.

\section{Discussion}

Energy allocation patterns are altered in the presence of fish kairomones, leading to changes in life history characteristics which enhance survival (Macháček, 1991; Stibor, 1992). In addition, the results presented here show that these life history changes are different for animals with different sizes at birth. Small neonates as well as large responded to the presence of fish kairomones by maturing earlier at a smaller size, and producing more but smaller offspring. The present results also show that size at birth influences the extent of the reaction to kairomones for size and age at maturity and number of eggs in the first clutch. Depending on the life history trait under investigation, either small or large neonates showed a greater difference between the fish and no-fish treatments. These differences are probably due to different trade-offs between life history traits which are not the same for neonates of various sizes.

In the no-fish treatment, small neonates matured later than large ones and at the same size, but produced fewer eggs which were approximately the same size. Apparently, part of the energy that was put into growth by the small neonates was put into reproduction by the large ones. Under these no-fish conditions large neonates therefore seem to be 

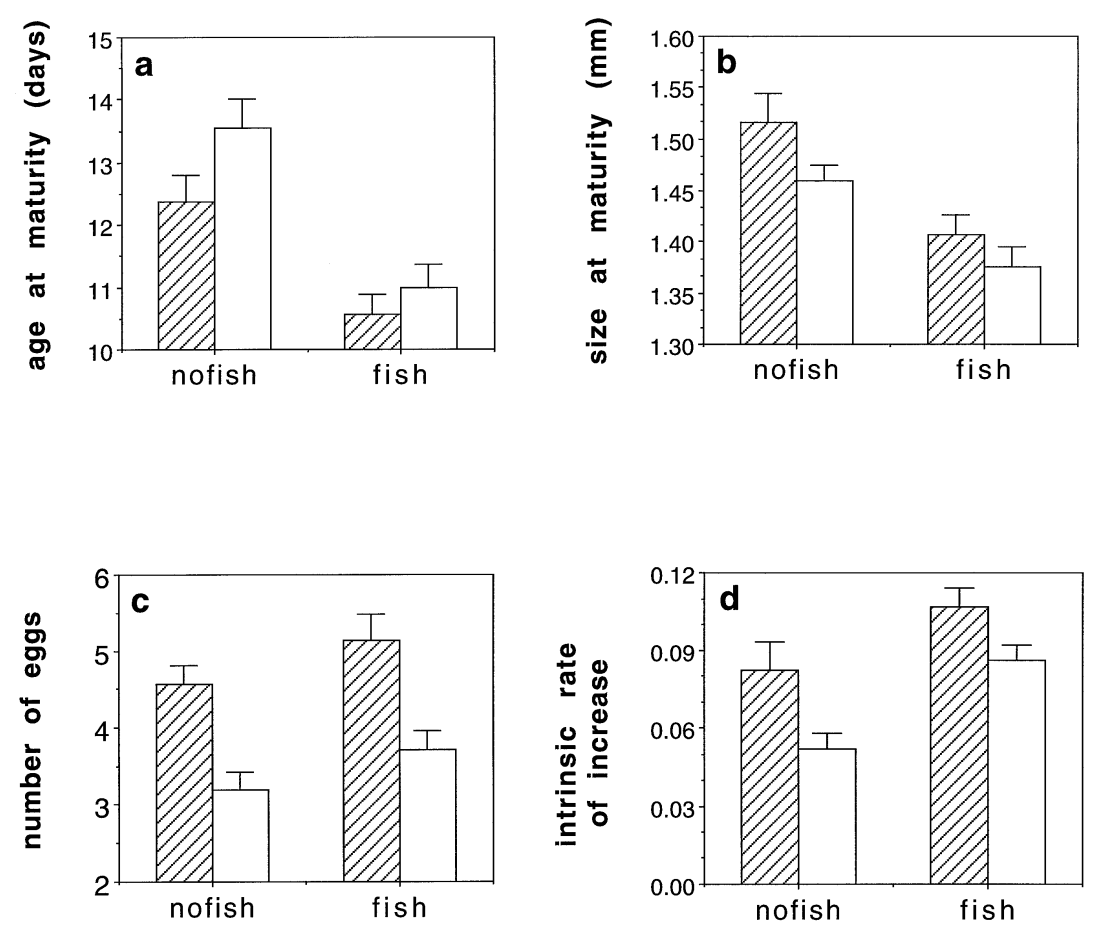

Fig. 2 (a) Age at maturity, (b) size at maturity, (c) number of eggs in the first clutch and (d) intrinsic rate of increase after one clutch in experiment 2. Error bars represent SEs. Shaded bars: high food concentration; open bars: low food concentration.

able to contribute more to reproduction. Generally, Daphnia produces larger neonates when fish predators are absent, although many small neonates are born when food conditions are very favourable. Predation by invertebrates is also an important factor influencing neonate size. When fish are absent many invertebrate predators become more abundant (Lynch, 1980). Boersma (1994), De Meester (1995) and Tollrian (1995) argue that in an environment with low vertebrate predation pressure, when Daphnia is able to produce several clutches, the production of large juveniles is favoured, because of both crowding (lack of food) and a higher likelihood of predation on juveniles by invertebrate predators. Indications for this are presented by Boersma (1994), who found that in a season with high juvenile fish biomass, densities of Daphnia of smaller size classes were relatively high whereas larger size classes were more predominant when fish densities were lower. Therefore, although many size classes of neonates can be produced in the absence of predatory fish, they will be larger than those born in their presence.

In the fish treatment, small and large neonates matured at the same age but, unexpectedly, the size at maturity of small neonates was larger than that of the large neonates. Both neonate groups produced about the same number of eggs but small neonates produced larger offspring. The fact that small neonates do not counterbalance the larger number of eggs by maturing very small or producing very small neonates, suggests that the life history response to kairomones is not simply the result of a shift in resource allocation patterns. It may present a stress factor influencing metabolic processes. Unfortunately, we do not yet know how kairomones operate. It seems quite probable that animals grown under kairomone conditions show a specific response to the food conditions. When food concentration is low, fewer but larger neonates occur and, because reproduction is low, extra precautions are needed to ensure survival. This might explain why large neonates matured at a smaller size than the small ones, or why they did not spend more energy on reproduction. When the life histories of small and large neonates in the presence of kairomones are compared, large neonates seem to spend less energy on growth and reproduction.

The present results also indicate that food concentration, within the range used here, does not markedly change the effect of kairomones since the difference between the fish and no-fish treatments was the same at both food concentrations. The

(C) 1997 Blackwell Science Ltd, Freshwater Biology, 37, 389-396 
exception is the intrinsic rate of increase (Fig. 2d). Machácek (1991) also observed no difference in kairomone effect between different food concentrations for the traits he investigated, but he did not calculate $r$. However, in Reede \& Ringelberg (1995) intrinsic rate of increase was significantly higher in the presence of kairomones at a food concentration of $0.57 \mathrm{mg} \mathrm{Cl}^{-1}$, but not at $1.14 \mathrm{mg} \mathrm{Cl}^{-1}$. In this experiment a significant difference occurred at $0.25 \mathrm{mg} \mathrm{Cl}^{-1}$ but not at $0.75 \mathrm{mg} \mathrm{Cl}^{-1}$ (Fig. 2d). Seemingly, somewhere between 0.57 and $0.75 \mathrm{mg} \mathrm{Cl}^{-1}$ the effect of fish kairomones on the intrinsic rate of increase diminishes. Although the differences between the food concentrations were not significant for three of the tested parameters, together they caused a significant effect on $r$.

In most studies, as in this one, daphnids from third and fourth clutches are used in experiments to avoid unwanted maternal effects. In the presence of predatory fish, however, the largest animals are most vulnerable to predation, so females may only survive to produce one or two clutches. Since these earlier neonates are smaller than the ones from later clutches, they will mature at smaller sizes and produce even smaller neonates (Lampert, 1993). Stibor (1992) found that second-generation daphnids, raised in an environment containing a predatorreleased chemical, had a smaller size at first reproduction than the first generation. Effects observed in the present experiment might be enhanced in the field because very large neonates will not occur when predatory fish are present.

In this study only one clone of the hybrid D. galeata $\times$ hyalina was used $(\mathrm{O} 2)$, which was assumed to be non-migratory (Reede \& Ringelberg, 1995) and which reacted more strongly to fish kairomones in a life history experiment than another clone which was assumed to be migratory. Both diel vertical migration and life history shifts are ways of avoiding predation and can be closely linked (De Meester, 1993, 1994). Each genotype may have its own way to respond to the presence of predators, either by adjusting behaviour or life history traits, or a combination the two (De Meester, Weider \& Tollrian, 1995). However, clones do not adjust both life history traits and behaviour maximally. Natural selection via predation or starvation resistance will determine which genotype is favoured at which time.

\section{Acknowledgments}

I wish to thank Luc de Meester and Bill DeMott for valuable comments which much improved the manuscript.

\section{References}

Barry M.J. (1995) The role of nutrition in regulation of predator-induced crests of Daphnia carinata. Freshwater Biology, 34, 229-239.

Boersma M. (1994) On the seasonal dynamics of Daphnia species in a shallow eutrophic lake. PhD thesis, University of Amsterdam, The Netherlands.

Cowgill U.M., Williams D.M. \& Esquivel J.B. (1984) Effects of maternal nutrition on fat content and longevity of neonates of Daphnia magna. Journal of Crustacean Biology, 4, 173-190.

De Meester L. (1993) Genotype, fish-mediated chemicals, and phototactic behavior in Daphnia magna. Ecology, 74, 1467-1474.

De Meester L. (1994) Life histories and habitat selection in Daphnia: divergent life histories of D. magna clones differing in phototactic behaviour. Oecologia, 97, 333341.

De Meester L. (1995) Life history characteristics of Daphnia magna clones in phototactic behaviour. Hydrobiologia, 307, 167-175.

De Meester L., Weider L.J. \& Tollrian R. (1995) Alternative antipredator defences and genetic polymorphism in a pelagic predator-prey system. Nature, 378, 483-485.

Ebert D. (1994) Functional resource allocation into few eggs: Daphnia as an example. Ecology, 75, 568-571.

Enserink L., Luttmer W. \& Maas-diepeveen H. (1990) Reproductive strategy of Daphnia magna affects the sensitivity of its progeny in acute toxicity tests. Aquatic Toxicology, 17, 15-25.

Glazier D.S. (1992) Effects of food, genotype, and maternal size and age on offspring investment in Daphnia magna. Ecology, 73, 910-926.

Guillard R.R.L. (1975) Culture of phytoplankton for feeding marine invertebrates. Culture of Marine Invertebrate Animals (eds W.L. Smith and M.H. Chanley), pp. 29-60. Plenum Press, New York,

Guisande C. \& Gliwicz Z.M. (1992) Egg size and clutch size in two Daphnia species grown at different food levels. Journal of Plankton Research, 14, 997-1007.

Hanazato T. \& Dodson S.I. (1995) Synergistic effects of low oxygen concentration, predator kairomone, and a pesticide on the cladoceran Daphnia pulex. Limnology and Oceanography, 40, 700-709. 
Lampert W. (1993) Phenotypic plasticity of the size at first reproduction in Daphnia: the importance of maternal size. Ecology, 74, 1455-1466.

Lynch M. (1980) The evolution of cladoceran life histories. Quarterly Review of Biology, 55, 23-42.

Macháček J. (1991) Indirect effect of planktivorous fish on the growth and reproduction of Daphnia galeata. Hydrobiologia, 225, 193-197.

Macháček J. (1995) Inducibility of life history changes by fish kairomone in various developmental stages of Daphnia. Journal of Plankton Research, 17, 1513- 1520.

Meyer J.S., Ingersoll C.G., McDonald L.L. \& Boyce M.S. (1986) Estimating uncertainty in population growth rates: jack-knife vs. bootstrap techniques. Ecology, 67, 1156-1166

Naylor C., Bradley M.C. \& Calow P. (1992) Effect of food ration on susceptibility of Daphnia magna Straus neonates to toxic substances. Aquatic Toxicology, 24, 75-82.

Parejko K. \& Dodson S.I. (1991) The evolutionary ecology of an antipredator reaction norm: Daphnia pulex and Chaoborus americanus. Evolution, 45, 1665-1674.

Reede T. (1995) Life history shifts in response to different levels of fish kairomones in Daphnia. Journal of Plankton Research, 17, 1661-1667.

Reede T. \& Ringelberg J. (1995) The influence of a fish exudate on two clones of the hybrid Daphnia galeata $\times$ hyalina. Hydrobiologia, 307, 207-212.

Sokal R.R. \& Rohlf F.J. (1981) Biometry, 2nd edn. W.H. Freeman, San Francisco, CA.

Stibor H. (1992) Predator induced life-history shifts in a freshwater cladoceran Oecologia, 92, 162-165.

Tessier A.J. \& Consolatti N.L. (1989) Variation in offspring size in Daphnia and consequences for individual fitness. Oikos, 56, 269-276.

Tessier A.J. \& Consolatti N.L. (1991) Resource quality and offspring quality in Daphnia. Ecology, 72, 468-478.

Tollrian R. (1995) Predator-induced morphological defences: costs, life history shifts, and maternal effects in Daphnia pulex. Ecology, 76, 1691-1705.

Urabe J. (1988) Effect of food concentration on the net production of Daphnia galeata: separate assessment of growth and reproduction. Bulletin of Plankton Society of Japan, 35, 159-174.

(Manuscript accepted 28 October 1996) 